\title{
Biological drugs in the treatment of atopic dermatitis - current recommendations of the Polish Dermatological Society, the Polish Society of Allergology, the Polish Pediatric Society and the Polish Society of Family Medicine
}

\author{
Roman J. Nowicki ${ }^{1}$, Magdalena Trzeciak ${ }^{1}$, Lidia Rudnicka², Jacek Szepietowski ${ }^{3}$, Marek Kulus ${ }^{4}$, Maciej Kupczyk ${ }^{5}$, \\ Agnieszka Mastalerz-Migas ${ }^{6}$, Jaroslaw Peregud-Pogorzelski ${ }^{7}$, Karina Jahnz-Różyk ${ }^{8}$, Joanna Narbutt ${ }^{9}$, \\ Magdalena Czarnecka-Operacz ${ }^{10}$, Rafał Czajkowski ${ }^{11}$, Elżbieta Grubska-Suchanek ${ }^{1}$, Dorota Krasowska ${ }^{12}$, \\ Beata Kręcisz ${ }^{13}$, Cezary Kowalewski ${ }^{14}$, Aleksandra Lesiak ${ }^{8}$, Małgorzata Olszewska², Zbigniew Samochocki², \\ Radosław Śpiewak ${ }^{15}$, Aleksandra Wilkowska ${ }^{1}$
}

\begin{abstract}
1Department of Dermatology, Venereology and Allergology, Medical University of Gdansk, Gdansk, Poland ${ }^{2}$ Department of Dermatology, Medical University of Warsaw, Warsaw, Poland

${ }^{3}$ Department of Dermatology, Venereology and Allergology, Medical University of Wroclaw, Wroclaw, Poland ${ }^{4}$ Department of Pediatric Pneumonology and Allergology, Medical University of Warsaw, Warsaw, Poland ${ }^{5}$ Department of Internal Medicine, Asthma and Allergy, Medical University of Lodz, Lodz, Poland ${ }^{6}$ Department of Family Medicine, Medical University of Wroclaw, Wroclaw, Poland

${ }^{7}$ Department of Pediatrics and Pediatric Oncology, Pomeranian Medical University, Szczecin, Poland

${ }^{8}$ Department of Internal Medicine, Pneumonology, Allergology and Clinical Immunology, Military Medical Institute, Warsaw, Poland ${ }^{9}$ Department of Dermatology, Pediatric and Oncological Dermatology, Medical University of Lodz, Lodz, Poland ${ }^{10}$ Department of Dermatology, Poznan University of Medical Sciences, Poznan, Poland

${ }^{11}$ Department of Dermatology and Venereology, "Ludwik Rydygier" Collegium Medicum in Bydgoszcz, Nicolaus Copernicus University inTorun, Poland

${ }^{12}$ Department of Dermatology, Venereology and Pediatric Dermatology, Medical University of Lublin, Lublin, Poland ${ }^{13}$ Department of Dermatology, Faculty of Medicine and Health Sciences, Jan Kochanowski University, Kielce, Poland

${ }^{14}$ Department of Dermatology and Immunodermatology, Medical University of Warsaw, Warsaw, Poland

${ }^{15}$ Department of Experimental Dermatology and Cosmetology, Jagiellonian University Medical College, Krakow, Poland
\end{abstract}

Adv Dermatol Allergol 2020; XXXVII (5): 617-624

DOI: https://doi.org/10.5114/ada.2020.100496

\begin{abstract}
Atopic dermatitis (AD) is secondary to genetic, immunological and microbiological disorders as well as epidermal barrier defects, which are the main targets of therapy. The disease proceeds with periodic exacerbations. Its development and course are influenced by numerous environmental and individual factors. In recent decades, in industrialized countries, there has been a threefold increase in the incidence of AD. There is also an increasing number of cases resistant to topical treatment. Effective treatment of AD should provide control of clinical symptoms, prevent exacerbations and improve the quality of life of patients. The multifactorial etiopathogenesis and various endotypes and phenotypes of AD justify the tendency to optimize and personalize the therapy. Currently, we recommend the use of dupilumab for the treatment of patients from 12 years of age with moderate and severe atopic dermatitis, who do not respond to topical treatment.
\end{abstract}

Key words: biological drugs, dupilumab, atopic dermatitis, therapeutic recommendations.

\section{Introduction}

Atopic dermatitis (ICD-10 L20) is a chronic disease that affects mainly children (10-20\%) and lasts until adulthood in about $1 / 5$ of this group [1]. The prevalence of $A D$ in adults has been estimated at 2.1-4.9\% [2]. In adulthood, the disease appears for the first time in one of four adult patients suffering from $\mathrm{AD}$ [3].

In over $60 \%$ of cases, there is an increased risk of developing atopic symptoms from other organs. Atopic der-

Address for correspondence: Prof. Roman J. Nowicki MD, PhD, Department of Dermatology, Venereology and Allergology, Medical University of Gdansk, 17 Smoluchowskiego St, 80-214 Gdansk, Poland, phone: +48 58584 40 10, e-mail: rnowicki@gumed.edu.pl Received: 19.10.2020, accepted: 2.11.2020. 
matitis is the first step in the atopic march, in the course of which the following may develop: food allergy (15\%), allergic rhinitis (34\%) and bronchial asthma (20-35\%). Very severe and persistent itching, hypersensitivity and visible skin inflammation and stigmatization significantly reduce the quality of life and are often the cause of anxiety, sleep disorders, absenteeism at school and work, social isolation and, consequently, depression, mental illness and even suicidal thoughts. Children with AD are more likely to develop abdominal obesity and arterial hypertension [4, 5].

Skin diseases associated with atopic dermatitis include ichthyosis (ichthyosis vulgaris), infectious diseases such as impetigo and herpetic eczema (eczema herpeticum), and immune diseases: alopecia areata and vitiligo (vitiligo). White dandruff (pityriasis alba) and keratosis pilaris are common in patients with AD [6, 7].

Cutaneous lymphoma may develop in adults with severe AD. Moreover, the relationship between AD and obesity, metabolic syndrome, cardiovascular diseases and osteoporosis has been described $[8,9]$.

\section{Biological drugs in atopic dermatitis}

Modern treatment of AD involves two groups of medicinal products: monoclonal bodies that act precisely to inhibit specific cytokines or their receptors, and agonists or antagonists of small molecules. Action of those latter is even broader. It is now known that, in addition to the defect in the epidermal barrier and disorders of the skin microbiota homeostasis, AD is immunologically characterized by abnormal activity of the Th2 cellular system expressed by excessive activity of cytokines such as IL4, IL13, cytokines secreted by Th17, Th22, Th1 lymphocytes and cytokines derived directly from the damaged epidermis (IL33, TSLP, IL25) [10-12]. Involvement of the Th2 cellular system seems to be the leading and ethnically or pheno-/endotypically independent factor. Which means it affects every patient with AD [13-15]. Hence, targeting the treatment to suppress the Th2 cellular system seems perfectly justified. In 2017, the FDA and EMA approved the drug dupilumab, the first biological drug in $A D$ for the treatment of its moderate and severe form.

\section{Dupilumab}

Dupilumab is a human lgG4 monoclonal antibody directed against the alpha subunit of the IL-4 receptor (IL$4 R \alpha$ ), thanks to which it blocks both IL-4 and IL-13. The current approval of the drug covers the treatment of moderate to severe $A D$ in patients aged 12 years and over.

\section{Efficacy of dupilumab therapy in children from 6 years of age, adolescents and adults}

The efficacy and safety of dupilumab as a monotherapy or administered concomitantly with topical glucocorticoids in adults were assessed in randomized, doubleblind, placebo-controlled phase $2 \mathrm{~b}$ trials in 452 patients, trials LIBERTY AD SOLO 1 and LIBERTY AD SOLO 2 in 1379 adult $A D$ patients, and 740 patients in the LIBERTY AD CHRONOS trial lasting up to 52 weeks [16-19]. The trials enrolled patients with a prior inadequate response to topical medications with severe to moderate AD as assessed by Investigator's Global Assessment (IGA) score $\geq 3$, eczema area and severity index (EASI), Eczema Area and Severity Index $\geq 16$ and Body Surface Area (BSA) $\geq 10 \%$. Dupilumab significantly improved the objective and subjective symptoms of AD and quality of life in patients with moderate to severe $A D$. A reduction in the SCORAD and EASI-75 scores, as well as a reduction in the severity of pruritus, anxiety/depression and sleep disorders have been observed in adults [16-21]. The drug is administered subcutaneously at the first dose of $600 \mathrm{mg}$, followed by the dose of $300 \mathrm{mg}$ s.c. every 2 weeks.

In the group of young people over 12 years of age, 251 patients with a mean age of $14.5 \pm 1.7$ years were randomized. Majority of these patients had other allergic diseases such as asthma, food allergy, and allergic rhinitis. Dupilumab significantly improved signs, symptoms and quality of life in adolescents with moderate to severe $A D$, with an acceptable safety profile. Effectiveness of the drug administered every 2 weeks was definitely higher than every 4 weeks [22]. Results were obtained and evaluated after 16 weeks of treatment. The drug is administered subcutaneously at the dose of $200 \mathrm{mg}$ in children weighing < $60 \mathrm{~kg}$ or $300 \mathrm{mg}$ for body weight $\geq 60 \mathrm{~kg}$, every 2 weeks or 300 mg every 4 weeks.

Data are available from the phase III clinical trial of dupilumab treatment with concomitant topical GCSs treatment in children aged 6-11 years with severe AD, with inadequately controlled topical treatment [23]. Three hundred sixty seven children were enrolled in the 16-week study. Dupilumab was effective and improved the quality of life of patients in this age group. Taking into account the effectiveness and side effects, the optimal dose was 300 mg every 4 weeks for children weighing less than $30 \mathrm{~kg}$ and 200 mg every 2 weeks for children weighing $30 \mathrm{~kg}$ or more [23]. Data from registries and descriptions of patients treated with dupilumab, in real life, apart from clinical trials, indicate comparable results to those obtained during clinical trials [24-29].

\section{Adverse reactions during dupilumab therapy in children from 6 years of age, adolescents and adults}

The most common adverse reaction observed in $22.1 \%$ of dupilumab users in adult clinical trials are conjunctivitis and blepharitis [30, 31]. In observations during the use of the drug outside clinical trials, this complication reaches the level of 38.2\% [26]. The suggested treatment is the use of artificial tears, drops with tGCS (fluorometholone $0.1 \%$ ), topical calcineurin inhibitors $(\mathrm{tCl})$ applied to the eyelids in moderate cases, and eye 
drops or ointments containing cyclosporin or tacrolimus in severe forms [31, 32].

Most cases of conjunctivitis are transient and can be successfully treated by continuing the therapy with dupilumab [29]. In terms of rates of infection, it was found that it was similar in adult patients receiving dupilumab and placebo. Dupilumab did not increase the risk of infection, nasopharyngitis, or upper respiratory tract infection, and the systemic use of anti-infective agents was lower in the dupilumab group. Fewer serious/severe bacterial infections and other non-herpetic skin infections were observed among patients using dupilumab [33]. Among the side effects with long-term use of dupilumab, paradoxical erythema of the face, décolleté and neck, usually after 10-39 weeks of using dupilumab, has been reported $[34,35]$. In the group of adolescents $>12$ years of age treated with dupilumab, a higher percentage of conjunctivitis and reactions at the injection site and lower rates of non-herpetic skin infections were observed [21]. Children with severe $A D$, aged 6-11 treated with dupilumab presented more frequent conjunctivitis and injection site reactions than the placebo group [23]. More research is needed in the pediatric group.

\section{Laboratory tests, monitoring during dupilumab therapy}

According to the evaluation of the results of available clinical trials conducted in the adult group, there is no need for laboratory monitoring during dupilumab therapy [36]. However, before and during dupilumab therapy (after 16 weeks and then every 3 months), we suggest performing basic tests (complete blood count with smear, AST, ALT, creatinine, urea, CRP). Before starting the therapy, we additionally recommend a pregnancy test in women of childbearing age, ECG and chest X-ray examinations (Table 1).

Table 1. Diagnostic procedures before and during the treatment with dupilumab

\begin{tabular}{l}
\hline Procedures before start of the therapy with dupilumab: \\
\hline General health assessment based on the medical history \\
Assessment of symptoms intensity with the EASI score \\
Blood cell count with smear \\
clinical chemistry: creatinine, urea, CRP, transaminases \\
(ALT, AST) \\
X-ray of the chest \\
ECG \\
Pregnancy test in women of childbearing potential \\
\hline Procedures after 16 weeks of the therapy, and then every \\
3 months ( \pm 7 days): \\
\hline General health assessment based on the medical history \\
Assessment of symptoms intensity with the EASI score \\
Blood cell count with smear \\
Clinical chemistry: creatinine, urea, CRP, transaminases \\
(ALT, AST)
\end{tabular}

Data on long-term safety in children and adolescents are time-limited and the size of the pediatric group indicates a need to extend the research in these age groups.

\section{Safety of long-term dupilumab therapy in children from 6 years of age, adolescents and adults}

Long-term safety of up to 3 years of use of dupilumab has been established for adults [37, 38]. In the group of children aged 12-17 years, safety data covers up to 52 weeks of treatment with dupilumab [39]. In this group, as well as in the group of 6-11-year-olds there are currently no results of studies lasting more than 1 year [23, 39].

\section{Dupilumab combined with other drugs used in atopic dermatitis}

Dupilumab should be combined with the daily use of emollients and, if necessary, may be combined with topical anti-inflammatory drugs [40].

\section{Recommendation}

The use of dupilumab is recommended in patients from 12 years of age with moderate to severe AD (EASI $\geq 16$, SCORAD $\geq 25$ ) who do not respond to local treatment. The duration of therapy depends on the resolution of the disease and the decision of the treating physician. Dupilumab may be self-administered subcutaneously by the patient or his caregivers at home (Table 2).

If there is no improvement after 16 weeks of dupilumab use (assessed as not achieving at least a 50\% reduction in the EASI score), treatment with this drug should be discontinued.

\section{Other biological drugs - monoclonal bodies in the treatment of atopic dermatitis}

Randomized, double-blind, placebo-controlled clinical trials are currently underway to evaluate the efficacy and safety of other biologics in adult patients with mild and severe AD. There are test results for monoclonal antibodies such as: tralokinumab, lebrikizumab (anti-IL-13),

Table 2. Dosage of dupilumab for subcutaneous administration to patients with atopic dermatitis

\begin{tabular}{lcc}
\hline Patients & Initial dose & $\begin{array}{c}\text { Subsequent } \\
\text { doses (every } \\
\text { 2 weeks) }\end{array}$ \\
\hline Adolescents from 12 to 17 years: & $200 \mathrm{mg}$ \\
\hline Below $60 \mathrm{~kg}$ & $\begin{array}{r}400 \mathrm{mg} \text { (two s.c. injections } \\
\text { a' } 200 \mathrm{mg})\end{array}$ \\
\hline 60 kg or more & $\begin{array}{r}600 \mathrm{mg} \text { (two s.c. injections } \\
\text { a' 300 mg) }\end{array}$ & $300 \mathrm{mg}$ \\
\hline Adults & $\begin{array}{r}600 \mathrm{mg} \text { (two s.c. injections } \\
\text { a' 300 mg) }\end{array}$ & $300 \mathrm{mg}$ \\
\hline
\end{tabular}


nemolizumab (anti-IL-31R $\alpha$ ), fezakinumab (anti-IL-22), etokimab (anti-IL-33) and tezepelumab (anti-TSLP).

\section{Lebrikizumab}

Lebrikizumab is an IL-13 binding monoclonal antibody. A randomized, placebo-controlled phase II clinical trial enrolled 209 adult patients with severe to moderate AD in whom topical glucocorticoids (tGCSs) were ineffective. The drug efficacy and safety were assessed after 12 weeks. The drug was administered to patients at the dose of 125 mg every 4 weeks - subcutaneously, and at the same time the use of tGCSs was allowed. Lebrikizumab led to significant clinical improvement and was well tolerated [41].

\section{Tralokinumab}

Tralokinumab is an IL-13 binding monoclonal antibody. The results of the phase $2 \mathrm{~b}$ clinical trial were published in 2019. The study included 204 adult patients with moderate and severe AD. The treatment was combined with tGCSs. Results summarized after 12 weeks of therapy indicated that the treatment with tralocanumab was associated with an early and sustained improvement in $A D$ symptoms with an acceptable safety and drug tolerance profile [42].

\section{Nemolizumab}

Given the pathogenesis of pruritus in AD, nemolizumab - an antibody directed against the IL-31 receptor - gave hope for improvement in this persistent symptom.

In a phase 2 study in 264 adult patients with severe and moderate AD who were not treated with tGCSs, 12 weeks of nemolizumab therapy brought a significant reduction in pruritus in treated patients. However, a limited number of patients enrolled in the study and a short evaluation period (12 weeks at the summary stage) did not allow the authors to draw conclusions about adverse effects [43]. Another phase $2 b$ study with nemolizumab $10 \mathrm{mg}, 30 \mathrm{mg}$ and $90 \mathrm{mg}$ administered subcutaneously every 4 weeks with 226 patients was summarized after 24 weeks of treatment. Nemolizumab produced a rapid and sustained improvement in cutaneous inflammation and itching with maximum efficacy seen with the $30 \mathrm{mg}$ dose. The safety profile of nemolizumab was assessed as acceptable [44]. The drug has also been evaluated in a long-term study in 264 patients with moderate to severe AD. Nemolizumab used for up to 64 weeks was effective and well tolerated. Similar responses in improvement of EASI-75 score were observed in the treated and placebo groups. However, the pruritus was significantly reduced in patients treated with the active drug [45].

\section{Fezakinumab}

Fezakinumab, an anti-IL-22 antibody, was evaluated in a Phase 2 a clinical trial in 60 patients with moderate to severe AD not controlled with tGCSs. The study ran for 20 weeks and results were summarized at week 12 . Fezakinumab was well tolerated and provided a longterm clinical improvement after the last drug administration [46].

\section{Etokimab}

Etokimab, an anti-IL-33 monoclonal IgG1 antibody. A phase 2 a study was conducted in 12 adult patients with moderate to severe AD. Patients received etokimab once. Rapid and sustained clinical improvement was observed. $83 \%$ of patients reached EASI50, and $33 \%$ reached EASI75, with a reduction in peripheral blood eosinophils on day 29 after dosing. These results confirm the role of IL-33 in modulation of the inflammatory cascade in atopic skin and confirm the therapeutic potential of IL-33 in the treatment of AD [47].

\section{Tezepelumab}

Tezepelumab is a monoclonal antibody directed against thymic stromal lymphopoietin (anti-TSLP). Considering the role of TSLP in the pathogenesis of $A D$, this cytokine derived from damaged keratinocytes raises high hopes as a therapeutic target. Results of the Phase $2 \mathrm{a}$ study involving 113 patients with moderate to severe AD who used tGCSs along with the study drug were summarized in weeks 12 and 16 of treatment with a subcutaneous dose of $280 \mathrm{mg}$ of the drug administered every 2 weeks. EASI50 was achieved by more patients treated with tezepelumab than with placebo. However, these differences were not statistically significant, although an increase in the proportion of responders at the week 16 of the study was observed [48].

\section{OX40 molecule antagonists}

OX40 is a costimulatory receptor on activated T cells (CD4). The fully human anti-OX40 monoclonal antibody was tested under the names KHK4083 and GBR830.

A phase 1 study evaluated the pharmacokinetics and immunogenicity of KHK4083 in 22 Japanese patients with moderate and severe AD [22]. The drug was administered at the dose of $10 \mathrm{mg} / \mathrm{kg}$ intravenously on days 1, 15 and 29 and patients were followed up to day 155. Multiple intravenous infusions of KHK4083 had an acceptable security profile. After the end of treatment with KHK4083, a sustained improvement in AD symptoms was observed [49]. It is interesting whether these results can be extrapolated to the European population in the face of hypotheses/theories of differentiation of AD endotypes also in the ethnic context. GBR 830 was also evaluated among US patients with moderate to severe $A D$ in a phase 2 a study that enrolled 64 patients. More than 40 people received the drug on days 1 and 29, and in 40 cases its effect was assessed in histopathological preparations. Two doses of GBR 830 given 4 weeks apart 
were well tolerated and induced resolution of tissue and clinical changes by day 71, confirming the therapeutic potential of this drug [50]. Results of studies with the monoclonal bodies described above refer to the second phase in adults. A clear limitation is the low size of study groups. Results of the third phase of this research may be expected soon.

\section{Agonists and antagonists of small molecules}

JAK-STAT inhibitors are a group of drugs that have been intensely studied in AD in recent years. These drugs are less selective than monoclonal bodies. They block the activation of Janus kinases, translocating the signal to the cell nucleus and, consequently, the transcription of many pro-inflammatory cytokines. Results of research completed so far are promising. These drugs are mainly used orally or topically.

\section{Abrocitinib}

Abrocitinib is a selective JAK 1 inhibitor. A Phase $2 b$, randomized, double-blind, placebo-controlled (RDBPC) study was conducted in 267 adult patients with moderate to severe $A D$ with insufficient response or contraindication to topical treatment. The results of the study, summarized after 12 weeks of treatment, showed that abrocitinib administered orally once daily was effective and well tolerated for short term use in adults with moderate to severe atopic dermatitis. However, additional studies were necessary to assess the long-term efficacy and safety of the drug [51]. This year, the results of the phase III JADE study were published [52]. Three hundred and eighty-seven adolescent patients from 12 years of age and adults with moderate and severe atopic dermatitis used the drug at the dose of $100 \mathrm{mg}$ or $200 \mathrm{mg}$. The study is still ongoing, but results summarized after 12 weeks of therapy showed the clinical effect of EASI75 for both doses of the drug. Oral once daily monotherapy with abrocitinib was effective and well tolerated in adolescents and adults with moderate to severe atopic dermatitis [52].

\section{Baricitinib}

Baricitinib is a selective inhibitor of JAK 1 and JAK2. A phase 2 study, RDBPC, was conducted in 124 adult patients with moderate to severe AD. The drug at the dose of $4 \mathrm{mg}$ and $2 \mathrm{mg}$ was used orally with the possibility of simultaneous application of tGCSs. After 16 weeks of the study, a significant reduction in the severity of clinical symptoms of $A D$ and an improvement in the quality of life was achieved. It is worth noting that improvement in clinical symptoms, including reduction of itching, was noted already in the first week of treatment in patients taking the drug at the dose of $4 \mathrm{mg}$. No serious adverse events were observed [53]. Results of the Phase III study have been published this year. The BREEZE-AD1 arm in- cluded 624 patients, and the BREEZE-AD2 arm included 615 patients with moderate and severe AD. Results of the study were collected after 16 weeks of treatment with baricitinib at doses of $1 \mathrm{mg}, 2 \mathrm{mg}$ and $4 \mathrm{mg}$. Baricitinib improved clinical signs and symptoms in patients with moderate to severe $A D$ and produced a rapid reduction of pruritus. The drug's safety profile was consistent with previous clinical studies with baricitinib in AD. The study is ongoing and new summaries of further results should be expected soon [54].

\section{Upadacitinib}

Upadacitinib is a selective Jak1 inhibitor. Results of the phase $2 \mathrm{~b}$ study, planned for 88 weeks in adult patients with moderate and severe $A D$, showed a significant effect of the drug on the reduction of itch from day 2 of the therapy [55]. A parallel study involving 167 adult patients with moderate to severe AD reported drug efficacy assessed using the EASI and NRS (pruritus severity scale), IGA scales at all doses, i.e. $7.5 \mathrm{mg}, 15 \mathrm{mg}$ and $30 \mathrm{mg}$ after 16 weeks of therapy. A dose-response relationship was observed for the efficacy of Spadacitinib; $30 \mathrm{mg}$ once daily produced the greatest clinical benefit. No dose limiting toxicity was observed [56].

\section{Tofacitinib}

Tofacitinib is a non-selective JAK 1/3 inhibitor. It was administered orally to 6 patients with moderate and severe atopic dermatitis, causing reduction of symptoms measured by the SCORAD scale without any adverse effects for up to 29 weeks of treatment, and it was applied topically in the form of $2 \%$ ointments $[57,58]$. Sixty-nine patients with mild to moderate $A D$ were enrolled in the phase 2a study. Results after 4 weeks of topical therapy showed a much greater clinical efficacy of the drug than placebo with an early onset of action (reduction of itch observed after 2 days of therapy) [58].

\section{Ruxolitinib}

The efficacy and safety of ruxolitinib (RUX) cream (a selective JAK1 and JAK2 inhibitor) at concentrations ranging from $0.15 \%$ to $1.5 \%$ was assessed in randomized, controlled phase 2 clinical trials of 307 adult patients with mild to moderate AD. Pruritus disappeared rapidly within 36 h of treatment, quality of life improved with a good drug tolerance [59].

\section{Delgocitinib}

Delgocitinib is an inhibitor of JAK 1,2,3 and TYK2. Results of the phase III study in 158 Japanese patients from 16 years of age with moderate to severe $A D$ are available. $0.5 \%$ delgocitinib ointment used over a period of 28 weeks was effective and well tolerated [60]. An earlier phase II study in 327 adult patients with moderate to severe adult $A D$ tested the drug at concentrations of $0.25 \%$, 
$0.5 \%, 1 \%, 3 \%$ of the ointment applied twice a day. Results showed a significant and rapid improvement in objective clinical symptoms and pruritus severity with a favorable safety profile [61]. Similarly, in the phase II study conducted in a group of 103 children aged 2 to 15 years with the use of delgocitinib at concentrations of $0.25 \%$ and $0.5 \%$, twice a day, an improvement in signs and symptoms, and good drug tolerance were demonstrated [62].

\section{Cerdulatinib and gusacitinib}

Cerdulatinib, a selective JAK and SYK (spleen tyrosine kinase) inhibitor, is currently in the phase 2 clinical trials using $0.4 \%$ topical gel and shows a reduction in clinical symptoms measured using the EASI scale [63].

Gusacitinib is another JAK/SYK kinase inhibitor in the phase $1 b$ RCT. It is used at the oral dose of $20 \mathrm{mg}$, $40 \mathrm{mg}$ and $80 \mathrm{mg}$ versus placebo for 4 weeks in $36 \mathrm{pa}$ tients with moderate to severe AD. Results after 4 weeks of treatment showed high effectiveness and quick onset of action [64].

\section{Conclusions}

Presented results of studies of new monoclonal antibodies and small molecules appear to be promising, but long-term safety and efficacy studies in larger groups of patients are still absent. Considering the complex etiopathogenesis of $A D$, endotypic and phenotypic differences, the prospect of having modern drugs for topical, oral and parental use in the therapeutic portfolio for both pediatric and adult groups in the near future is encouraging

Currently, for the treatment of patients from 12 years of age with moderate to severe AD who do not respond to topical treatment, we recommend the use of dupilumab. Treatment should be initiated by physicians experienced in the diagnosis and treatment of $A D$.

\section{Conflict of interest}

The authors declare no conflict of interest.

\section{References}

1. Bylund S, von Kobyletzki LB, Svalstedt M, Svensson A. Prevalence and incidence of atopic dermatitis: a systematic review. Acta Derm Venereol 2020; 100: adv00160.

2. Barbarot S, Auziere S, Gadkari A, et al. Epidemiology of atopic dermatitis in adults: results from an international survey. Allergy 2018; 73: 1284-93.

3. Vakharia PP, Silverberg JI. Adult-onset atopic dermatitis: characteristics and management. Am J Clin Dermatol 2019; 20: 771-9.

4. Czarnowicki T, Krueger JG, Guttman-Yassky E. Novel concepts of prevention and treatment of atopic dermatitis through barrier and immune manipulations with implications for the atopic march. J Allergy Clin Immunol 2017; 139: 1723-34.
5. Silverberg JI, Becker L, Kwasny M, et al. Central obesity and high blood pressure in pediatric patients with atopic dermatitis. JAMA Dermatol 2015; 151: 144-52.

6. Sun D, Ong PY. Infectious complications in atopic dermatitis. Immunol Allergy Clin North Am 2017; 37: 75-93.

7. Fenner J, Silverberg NB. Skin diseases associated with atopic dermatitis. Clin Dermatol 2018; 36: 631-40.

8. Paller A, Jaworski JC, Simpson EL, et al. Major comorbidities of atopic dermatitis: beyond allergic disorders. Am J Clin Dermatol 2018; 19: 821-38.

9. Silverberg JI, Simpson EL. Association between severe eczema in children and multiple comorbid conditions and increased healthcare utilization. Pediatr Allergy Immunol 2013; 24: 476-86.

10. Paller AS, Kong HH, Seed P, et al. The microbiome in patients with atopic dermatitis. J Allergy Clin Immunol 2019; 143: 26-35.

11. Palmer CN, Irvine AD, Terron-Kwiatkowski A. Common lossof-function variants of the epidermal barrier protein filaggrin are a major predisposing factor for atopic dermatitis. Nat Genet 2006; 38: 441-6,

12. Weidinger S, Beck LA, Bieber T, et al. Atopic dermatitis. Nat Rev Dis Primers 2018; 4: 1.

13. Noda S, Suárez-Farińas M, Ungar B, The Asian atopic dermatitis phenotype combines features of atopic dermatitis and psoriasis with increased TH17 polarization. J Allergy Clin Immunol 2015; 136: 1254-64.

14. Brunner PM, Israel A, Zhang N, et al. Early-onset pediatric atopic dermatitis is characterized by Th2, Th22, Th17-centered inflammation and lipid alterations. J Allergy Clin Immunol 2018; 141: 2094-106.

15. Czarnowicki T, He H, Krueger JG, Guttman-Yassky E. Atopic dermatitis endotypes and implications for targeted therapeutics. J Allergy Clin Immunol 2019; 143: 1-11.

16. Beck LA, Thaci D, Hamilton JD, et al. Dupilumab treatment in adults with moderate-to-severe atopic dermatitis. N Engl J Med 2014; 371: 130-9.

17. Thaci D, Simpson EL, Beck LA, et al. Efficacy and safety of dupilumab in adults with moderate-to-severe atopic dermatitis inadequately controlled by topical treatments: a randomised, placebo-controlled, dose-ranging phase $2 \mathrm{~b}$ trial. Lancet 2016; 387: 40-52.

18. Thaçi D, L Simpson E, Deleuran M, et al. Efficacy and safety of dupilumab monotherapy in adults with moderate-to-severe atopic dermatitis: a pooled analysis of two phase 3 randomized trials (LIBERTY AD SOLO 1 and LIBERTY AD SOLO 2). J Dermatol Sci 2019; 94: 266-75.

19. Blauvelt A, de Bruin-Weller M, Gooderham M, et al. Longterm management of moderate-to-severe atopic dermatitis with dupilumab and concomitant topical corticosteroids (LIBERTY AD CHRONOS): a 1-year, randomised, doubleblinded, placebo-controlled, phase 3 trial. Lancet 2017; 389: 2287-303.

20. Simpson EL, Akinlade B, Ardeleanu M. Two phase 3 trials of dupilumab versus placebo in atopic dermatitis. N Engl I Med 2017; 376: 1090-1.

21. Barbarot S, Wollenberg A, Silverberg Jl, et al. Dupilumab provides rapid and sustained improvement in SCORAD outcomes in adults with moderate-to-severe atopic dermatitis: combined results of four randomized phase 3 trials. J Dermatolog Treat 2020; doi: 10.1080/09546634.2020.1750550.

22. Simpson EL, Paller AS, Siegfried EC, et al. Efficacy and safety of dupilumab in adolescents with uncontrolled moderate to 
severe atopic dermatitis: a phase 3 randomized clinical trial. JAMA Dermatol 2019; 156: 44-56.

23. Paller AS, Siegfried EC, Thaçi D, et al. Efficacy and safety of dupilumab with concomitant topical corticosteroids in children 6 to 11 years old with severe atopic dermatitis: a randomized, double-blinded, placebo-controlled phase 3 trial J Am Acad Dermatol 2020; 83: 1282-93.

24. Ariëns LFM, van der Schaft J, Bakker DS, et al. Dupilumab is very effective in a large cohort of difficult-to-treat adult atopic dermatitis patients: first clinical and biomarker results from the BioDay registry. Allergy 2020; 75: 116-26.

25. Jang DH, Heo SJ, Jung HJ, et al. Retrospective study of dupilumab treatment for moderate to severe atopic dermatitis in Korea: efficacy and safety of dupilumab in real-world practice. J Clin Med 2020; 9: 1982.

26. Fargnoli MC, Esposito M, Ferrucci S, et al. Real-life experience on effectiveness and safety of dupilumab in adult patients with moderate-to-severe atopic dermatitis I Dermatol Treat 2019; doi: 10.1080/09546634.2019.1682503.

27. Abraham S, Haufe E, Harder I, et al. Implementation of dupilumab in routine care of atopic eczema: results from the German national registry TREATgermany. Br J Dermatol 2020; 183: 382-4.

28. Faiz S, Giovannelli J, Podevin C, et al. Effectiveness and safety of dupilumab for the treatment of atopic dermatitis in a real-life French multicenter adult cohort. J Am Acad Dermatol 2019; 81: 143-51.

29. de Wijs LEM, Bosma AL, Erler NS, et al. Effectiveness of dupilumab treatment in 95 patients with atopic dermatitis: daily practice data. J Dermatol 2020; 182: 418-26.

30. Akinlade B, Guttman-Yassky E, de Bruin-Weller M, et al. Conjunctivitis in dupilumab clinical trials. Br I Dermatol 2019; 181: 459-73.

31. Wollenberg A, Ariens L, Thurau S, et al. Conjunctivitis occurring in atopic dermatitis patients treated with dupilumabclinical characteristics and treatment. J Allergy Clin Immunol Pract 2018; 6: 1778-80e1771.

32. Thyssen JP, de Bruin-Weller MS, Paller AS, et al. Conjunctivitis in atopic dermatitis patients with and without dupilumab therapy - international eczema council survey and opinion. J Eur Acad Dermatol Venereol 2019; 33: 1224-31.

33. Eichenfield LF, Bieber T, Beck LA, et al. Infections in dupilumab clinical trials in atopic dermatitis: a comprehensive pooled analysis. Am J Clin Dermatol 2019; 20: 443-56.

34. de Wijs LEM, Nguyen NT, Kunkeler ACM, et al. Clinical and histopathological characterization of paradoxical head and neck erythema in patients with atopic dermatitis treated with dupilumab: a case series. Br J Dermatol 2020; 183: 745-9.

35. Soria A, Du-Thanh A, Seneschal J, et al. Development or exacerbation of head and neck dermatitis in patients treated for atopic dermatitis with dupilumab. JAMA Dermatol 2019; 155: 1312-5.

36. Wollenberg A, Beck LA, Blauvelt A, et al. Laboratory safety of dupilumab in moderate-to-severe atopic dermatitis: results from three phase III trials (LIBERTY AD SOLO 1, LIBERTY AD SOLO 2, LIBERTY AD CHRONOS). Assessing the need for routine safety testing for patients being treated with dupilumab for moderate-to-severe atopic dermatitis. J Dermatol 2020; 182: e186-209.

37. Beck LA, Thaçi D, Deleuran M, et al. Dupilumab provides favorable safety and sustained efficacy for up to 3 years in an open-label study of adults with moderate-to-severe atopic dermatitis. Am J Clin Dermatol 2020; 21: 567-77.
38. Deleuran M, Thaçi D, Beck LA, et al. Dupilumab shows longterm safety and efficacy in patients with moderate to severe atopic dermatitis enrolled in a phase 3 open-label extension study. I Am Acad Dermatol 2020; 82: 377-88.

39. Cork MJ, Thaçi D, Eichenfield LF, et al. Dupilumab in adolescents with uncontrolled moderate-to-severe atopic dermatitis: results from a phase lla open-label trial and subsequent phase III open-label extension. Br J Dermatol 2020; 182: 85-96.

40. de Bruin-Weller M, Thaçi D, Smith C, et al. Dupilumab with concomitant topical corticosteroid treatment in adults with atopic dermatitis with an inadequate response or intolerance to ciclosporin A or when this treatment is medically inadvisable: a placebo-controlled, randomized phase III clinical trial (LIBERTY AD CAFÉ). Br J Dermatol 2018; 178: 1083-101.

41. Simpson EL, Flohr C, Eichenfield LF, et al. Efficacy and safety of lebrikizumab (an anti-IL-13 monoclonal antibody) in adults with moderate-to-severe atopic dermatitis inadequately controlled by topical corticosteroids: a randomized, placebo-controlled phase II trial (TREBLE). J Am Acad Dermatol 2018; 78: 863-71e811.

42. Wollenberg A, Howell MD, Guttman-Yassky E, et al. Treatment of atopic dermatitis with tralokinumab, an anti-IL-13 mAb. J Allergy Clin Immunol 2019; 143: 135-41.

43. Ruzicka T, Hanifin JM, Furue M, et al. Anti-interleukin-31 receptor A antibody for atopic dermatitis. N Engl I Med 2017; 376: 826-35.

44. Silverberg JI, Pinter A, Pulka G, et al. Phase 2B randomized study of nemolizumab in adults with moderate-to-severe atopic dermatitis and severe pruritus. J Allergy Clin Immunol 2020; 145: 173-82.

45. Kabashima K, Furue M, Hanifin JM, et al. Nemolizumab in patients with moderate-to-severe atopic dermatitis: randomized, phase II, long-term extension study. J Allergy Clin Immunol 2018; 142: 1121-30e7.

46. Guttman-Yassky E, Brunner PM, Neumann AU, et al. Efficacy and safety of fezakinumab (an IL-22 monoclonal antibody) in adults with moderate-to-severe atopic dermatitis inadequately controlled by conventional treatments: a randomized, double-blind, phase 2a trial. J Am Acad Dermatol 2018; 78: 872-81.

47. Chen YL, Gutowska-Owsiak D, Hardman CS, et al. Proof-ofconcept clinical trial of etokimab shows a key role for IL-33 in atopic dermatitis pathogenesis. Sci Transl Med 2019; 11: eaax2945.

48. Simpson EL, Parnes JR, She D, et al Tezepelumab, an antithymic stromal lymphopoietin monoclonal antibody, in the treatment of moderate to severe atopic dermatitis: a randomized phase 2a clinical trial. J Am Acad Dermatol 2019; 80: 1013-21.

49. Nakagawa H, lizuka H, Nemoto O, et al. Safety, tolerability and efficacy of repeated intravenous infusions of KHK4083, a fully human anti-OX40 monoclonal antibody, in Japanese patients with moderate to severe atopic dermatitis. J Dermatol Sci 2020; S0923-1811(20)30201-2.

50. Guttman-Yassky E, Pavel AB, Zhou L, et al. GBR 830, an antiOX40, improves skin gene signatures and clinical scores in patients with atopic dermatitis. J Allergy Clin Immunol 2019; 144: 482-93.

51. Gooderham MJ, Bissonnette R, Beebe JS, et al. Efficacy and safety of oral janus kinase 1 inhibitor abrocitinib for patients with atopic dermatitis: a phase 2 randomized clinical trial. JAMA Dermatol 2019; 155: 1371-9. 
52. Simpson EL, Sinclair R, Forman S, et al. Efficacy and safety of abrocitinib in adults and adolescents with moderateto-severe atopic dermatitis (JADE MONO-1): a multicentre, double-blind, randomised, placebo-controlled, phase 3 trial. Lancet 2020; 396: 255-66.

53. Guttman-Yassky E, Silverberg JI, Nemoto O, et al. Baricitinib in adult patients with moderate-to-severe atopic dermatitis: a phase 2 parallel, double-blinded, randomized placebocontrolled multiple-dose study. J Am Acad Dermatol 2019; 80: 913-21.e9.

54. Simpson EL, Lacour JP, Spelman L, et al. Baricitinib in patients with moderate-to-severe atopic dermatitis and inadequate response to topical corticosteroids: results from two randomized monotherapy phase III trials. Br J Dermato 2020; 183: 242-55.

55. Beck L, Hong C, Hu X, et al. Upadacitinib effect on pruritus in moderate-to-severe atopic dermatitis: from a phase $2 b$ randomized, placebo-controlled trial. Ann Allergy Asth Immunol 2018; 121: S21.

56. Guttman-Yassky E, Thaçi D, Pangan AL, et al. Upadacitinib in adults with moderate to severe atopic dermatitis: 16-week results from a randomized, placebo-controlled trial. J Allergy Clin Immunol 2020; 145: 877-84.

57. Levy LL, Urban J, King BA. Treatment of recalcitrant atopic dermatitis with the oral Janus kinase inhibitor tofacitinib citrate. J Am Acad Dermatol 2015; 73: 395-9.

58. Bissonnette R, Papp KA, Poulin Y, et al. Topical tofacitinib for atopic dermatitis: a phase Ila randomized trial. Br J Dermatol 2016; 175: 902-11.

59. Kim BS, Sun K, Papp K, et al. Effects of ruxolitinib cream on pruritus and quality of life in atopic dermatitis: results from a phase 2, randomized, dose-ranging, vehicle- and activecontrolled study. J Am Acad Dermatol 2020; 82: 1305-13.

60. Nakagawa H, Nemoto O, Igarashi A, et al. Delgocitinib ointment, a topical Janus kinase inhibitor, in adult patients with moderate to severe atopic dermatitis: a phase 3, randomized, double-blind, vehicle-controlled study and an openlabel, long-term extension study. J Am Acad Dermatol 2020; 82: 823-31.

61. Nakagawa H, Nemoto O, Igarashi A, et al. Efficacy and safety of topical JTE-052, a Janus kinase inhibitor, in Japanese adult patients with moderate-to-severe atopic dermatitis: a phase II, multicentre, randomized, vehicle-controlled clinical study. Br J Dermatol 2018; 178: 424-32.

62. Nakagawa $\mathrm{H}$, Nemoto O, Igarashi A, et al. Phase 2 clinical study of delgocitinib ointment in pediatric patients with atopic dermatitis. J Allergy Clin Immunol 2019; 144: 1575-83.

63. Piscitelli S, Lee J, McHale K, et al. Cerdulatinib (DMVT-502), a novel, topical dual Janus kinase/spleen tyrosine kinase inhibitor, improves the cellular and molecular cutaneous signature in patients with atopic dermatitis. Exp Dermatol 2018; 27: S2.

64. Bissonnette R, Maari C, Forman S, et al. The oral Janus kinase/spleen tyrosine kinase inhibitor ASN 002 demonstrates efficacy and improves associated systemic inflammation in patients with moderate-to-severe atopic dermatitis: results from a randomized double-blind placebocontrolled study. Br J Dermatol 2019; 181: 733-42. 